\title{
Numerical Prediction of Rose Growth
}
E. Bernsen
O. Bokhove
D. van der Sar
Department of Physics
Dept. of Applied Mathematics
Phytocare
University of Utrecht
University of Twente
Berkel en Rodenrijs
Utrecht, The Netherlands
Enschede, The Netherlands
The Netherlands
E.Bernsen@phys.uu.nl
o.bokhove@math.utwente.nl
dick.vdsar@phytocare.nl

Keywords: Rose growth model, stem density function, leaf photosynthesis

\begin{abstract}
A new mathematical model is presented for the prediction of rose growth in a greenhouse. Given the measured ambient environmental conditions, the model consist of a local photosynthesis model, predicting the photosynthesis per unit leaf area, coupled to a global greenhouse model, which predicts the mass production of the rose crop per unit area of greenhouse in time. The numerical implementation of this mathematical rose model is validated against harvest data of the rose variety "Red Berlin" from January $1^{\text {st }} 2001$ through to January $20^{\text {th }} \mathbf{2 0 0 2}$. Global trends are predicted reasonably well, and larger deviations between model and data in certain weeks are due to actual pest and fertigation mismatches not present in the model. We hypothesize that further improvements in the relation between the change in the crop's mass and the total photosynthesis, the dependence of the sprouting sites on the total photosynthesis and the parameter fitting can strengthen the leading-order model considered.
\end{abstract}

Memorandum 1803

Mathematics Subject Classification: 9206, 92B05, 9208

\section{INTRODUCTION}

We present numerical predictions for rose growth in a greenhouse based on an extension of the mathematical rose growth model developed in Bokhove et al. (2002). That rose model predicts the amount of harvested rose crop given measured environmental conditions — being light intensity, temperature, ambient $\mathrm{CO}_{2}$ concentration and relative humidity - in the greenhouse and available as an extensive time-dependent data set. The goal of our article is to test this leading-ordermodel for rose growth numerically against measurements of harvest rates for the given environmental conditions, identify its shortcomings and formulate improvements. The ultimate aim of rose growth predictions is to aid in optimizing rose growth by active control of the environmental greenhouse conditions. Our rose model consists of two parts: (a) a local description of the photosynthetic rate per unit or infinitesimal leaf area taken from Harley et al. (1992), and Kim and Lieth (2001, 2003); and, 
(b) a global description of the crop per unit area in the greenhouse using a more convenient formulation of the model originally developed in Bokhove et al. (2002).

The rose plants in the greenhouse are separated into two parts: the crop and the bush. The crop consists of the rose stems, which are harvested, and lie above height $h=l_{0}$. The bush is below the crop and it contains the body of rose plants supporting each individual stem. The bush is not harvested and lies between heights $0 \leq h \leq l_{0}$.

The global part of the model is based on the following assumptions (Bokhove et al., 2002), which arise from the growers' experiences with greenhouse rose growth: (i) Roses are to leading order unselfish in that all biomass gained through photosynthesis is equally distributed over all stems in a rose plant.

(ii) Stems grow vertically.

(iii) Stems start growing at height $h=l_{0}$ with an adjusted "length" $l=l_{0}$, and are harvested continuously when they reach the (adjusted) "length" $l=l_{c u t}$. Note that these "lengths" are measured from the bottom of the bush, i.e. from height $h=0$, to accommodate the mathematical modeling. When a stem is harvested, $l$ is reduced to $l=l_{0}$ : the actual stem is removed.

(iv) The number of sprouting sites at height $h=l_{0}$ is a continuous, real number when averaged per unit area of greenhouse, and proportional to the total photosynthesis but limited by a weighted maximum.

(v) New leaves appear at the stem top and are thus the youngest stem leaves.

(vi) Mass and leaf area are proportional to the stem length and uniformly distributed along the stem.

The evolution of rose growth now depends on the evolution of a stem density function $D$ which is related to the number of rose stems per unit area of greenhouse. Due to the photosynthesis the roses grow and the stem density function changes. The purpose of the global part of the model is to couple the evolution of the stem density function to a local model for the photosynthetic rate - here chosen to be the model of Kim and Lieth $(2001,2003)$. This results in a non-linear integro-partialdifferential equation for the stem density function. The harvest rate, number of stems and total mass (per unit area of greenhouse) can be related to integrals of this stem density function over leaf area and length.

Our mathematical model resembles Dayan et al.'s (1993ab) model in its use of this stem density function and the age of a stem to predict harvest rates, but differs in its elaborate calculation of the total photosynthesis based on the local photosynthesis at each infinitesimal leaf area and the stem density function. The harvest rate, total mass of crop and the number of stems in the green house are readily related to this stem density function, which is very appealing. To explore the potential of the novel approach, we restrict ourselves to a leading-order model in which $D$ only depends on $l$ and $t$ such that $D=D(l, t)$. More complex and perhaps more realistic dependencies are left as future work.

Several unknown proportionality constants are introduced in the mathematical model presented in the next section, which are partly estimated using direct observations and partly determined in a fit of the model to harvest measurements of 
the rose variety "Red Berlin" and environmental conditions in the greenhouse from January $1^{\text {st }} 2001$ to January $20^{\text {th }} 2002$. We compare predictions over this period of the full and a simplified model with fits of two key parameters using the total and half of the harvest data. Finally, we draw conclusions about the strengths and weaknesses of the model and recommend future research alleys.

\section{MATHEMATICAL MODEL}

The main variables in our model are the stem density function $D=D(l, t)$ (expressed in inverse cubic meters, $\mathrm{m}^{-3}$ ) and stem age function $A=A(l, t)$ (expressed in seconds, s), as function of stem length $l$ and time $t$. The number of stems of length between $l$ and $l+\mathrm{d} l$ per square meter of greenhouse is given by $D(l, t) \mathrm{d} l$. According to the unselfishness assumption (i) all stems have the same rate of growth, $v(t)$, and hence there is a one-to-one relation between stem height and stem age. The age of a stem of length $l$ is given by $A(l, t)$ and is by assumption (v) the age of the oldest leaves at $h=l_{0}$. Since $D$ and $A$ are advected by the same growth velocity, $v$, we obtain the following partial differential equations for $D$ and $A$ (cf. ideas in Dayan et al., 1993ab):

$$
\begin{aligned}
& \partial_{t} D(l, t)+v(t) \partial_{l} D(l, t)=0 \\
& \partial_{t} A(l, t)+v(t) \partial_{l} A(l, t)=1
\end{aligned}
$$

with $\partial_{l}=\partial / \partial l, \partial_{t}=\partial / \partial t$ and $l_{0}<l<l_{\text {cut }}$ following assumption (iii). The death rate of stems is assumed negligible. Stems of length $l=l_{\text {cut }}$ are harvested and cut down to a length of $l=l_{0}$. The harvest rate per unit area is therefore given by:

$$
H(t)=K_{3} v(t)\left(l_{\text {cut }}-l_{0}\right) D\left(l_{\text {cut }}, t\right)
$$

with $K_{3}$ the mass of rose per unit length of stem. The units of all parameters are found in Table 1. The total mass $M(t)$ and total number $N(t)$ of stems, both per square meter, are related to the first and zeroth moments of the stem density function, as follows

$$
M(t)=K_{3} \int_{l_{0}}^{l_{\text {cut }}}\left(l-l_{0}\right) D(l, t) \mathrm{d} l \quad \text { and } \quad N(t)=\int_{l_{0}}^{l_{c u t}} d(l, t) \mathrm{d} l .
$$

Using (1a), (2) and (3), the change of mass per square meter of greenhouse can be derived through differentiation and integration by parts

$$
\frac{\mathrm{d} M}{\mathrm{~d} t}+H(t)=K_{3} v(t) N(t)=K_{1} P_{n e t}(t),
$$

see also Bernsen et al. (2006), which is assumed proportional to the net photosynthetic rate $P_{n e t}(t)$ in (4), with $K_{1}$ a proportionality constant representing the amount of stem mass production per unit of photosynthesis. This simplification is a leading-order approach in which photosynthesis is assumed to be the principal driving mechanism. Mass loss or gain by transport of nutrients and water through 
the roots and leaves are therefore slaved directly to this net photosynthesis. This is a simplifying but also potentially weakening modeling strategy. Hence, from (4) we obtain an expression for the advection or growth velocity

$$
v(t)=K_{1} P_{n e t} /\left(K_{3} N\right) .
$$

The net photosynthesis consists of the photosynthesis in the crop and the photosynthesis in the bush

$$
P_{\text {net }}(t)=P_{\text {crop }}(t)+P_{\text {bush }}(t) .
$$

By integration over leaf age $a$ and height $h$ the global photosynthesis $P_{\text {crop }}(t)$ can be derived from the local photosynthesis $P_{\text {leaf }}(a, I(h), t)$ per square meter of leaf of age $a$ and with light intensity $I=I(h, t)$ at height $h$ (see Kim and Lieth, 2001, 2003). The local photosynthesis model also depends on ambient temperature, $\mathrm{CO}_{2}$ concentration and relative humidity, which are measured frequently. Full details of these dependencies are found in Kim and Lieth (2001) and Bokhove et al. (2002). We emphasize, however, that the global model can in principle be coupled to any other and better local photosynthesis model. Using this local model we can derive (a technical argument detailed in Bernsen et al., 2006, and Bokhove et al., 2002) the total photosynthesis in the crop as

$$
P_{\text {crop }}(t)=K_{4} \int_{l_{0}}^{l_{c u t}} \int_{l_{0}}^{l_{0}+l_{\text {cut }}-h} D\left(l-l_{0}+h, t\right) P_{\text {leaf }}(A(l, t), I(h, t), t) \mathrm{d} l \mathrm{~d} h
$$

with $K_{4}$ the leaf area of a stem per unit of length. The light at height $h$ is assumed to satisfy the following equation (cf. Kim and Lieth, 2001)

$$
\partial_{h} I(h, t)=K_{6} \rho(h, t) I(h, t) \quad \text { with } \quad I\left(l_{c u t}, t\right)=I_{0}(t),
$$

$\partial_{h}=\partial / \partial_{h}, I_{0}(t)$ the light intensity entering the greenhouse and $K_{6}$ the light absorption coefficient, where $\rho(h, t) \mathrm{d} h$ is the total leaf area per square meter of greenhouse at height between $h$ and $h+\mathrm{d} h$. Solving (8) results in the following expression of the light intensity

$$
I(h, t)=I_{0} \exp \left(-K_{6} \int_{h}^{l_{c u t}} \rho\left(h^{\prime}, t\right) \mathrm{d} h^{\prime}\right) \quad \text { with } \quad \rho(h, t)=K_{4} \int_{h}^{l_{c u t}} D(l, t) \mathrm{d} l,
$$

where we recognize $\int_{h}^{l_{c u t}} \rho\left(h^{\prime}, t\right) \mathrm{d} h^{\prime}$ as the cumulative leaf area index of the crop going down from the top of the crop (cf. expression (10) in Kim and Lieth, 2001). For the photosynthesis in the bush the following simple approximation is used

$$
P_{\text {bush }}(t)=K_{8} P_{\text {leaf }}\left(a_{\text {bush }}, I\left(l_{0}\right), t\right)
$$

with $a_{b u s h}$ the average age of leaves in the bush, $I\left(l_{0}\right)$ the light intensity reaching the bush and $K_{8}$ a constant representing the total leaf area in the bush per square meter of greenhouse. The system (1a) and (1b) forms thus a system of integro-partial 
differential equations, since the advection velocity $v$ defined in (5) depends through the net photosynthesis $P_{n e t}$ on integrals of $D$ and $A$.

Finally, to complete the model we need to specify boundary and initial conditions for the variables $D$ and $A$ governed by partial differential equations (1a) and (1b). In the case that $v>0$, which is usually the case, assumption (iv) is used to get the following condition for the density of newly created stems

$$
D\left(l_{0}, t\right)=K_{2} P_{\text {net }}(t)(S-N(t)) / S
$$

with $S$ the maximum number of sprouting sites per square meter of greenhouse and $K_{2}$ a constant. The stem density function is thus taken to be proportional to the instantaneous photosynthesis limited by a maximum number $S$ of sprouting sites. Newly created stems are of age zero, hence we have $A\left(l_{0}, t\right)=0$. If $v<0$ rose stems are shrinking and we have to specify $D$ and $A$ at $l=l_{\text {cut }}$. Since stems of length $l_{\text {cut }}$ are cut off we specify for the stem density function: $D\left(l_{c u t}, t\right)=0$. It appears that we can take anything for $A\left(l_{c u t}, t\right)$, including zero, since $D(l, t)=0$ for all $l$ influenced by the boundary conditions for $A$ at $l_{c u t}$.

\section{METHOD}

For the period January $1^{\text {st }} 2001$ to January $20^{\text {th }} 2002$ the harvest in a greenhouse is measured as well as the ambient environmental conditions: the relative humidity $R_{H}$, the light intensity $I_{0}$ entering the greenhouse, the ambient temperature $T_{a}$ and $\mathrm{CO}_{2}$ concentration $\mathrm{C}_{a}$. These four measured climate variables, together with initial conditions for $D$ and $A$, are used as input for the mathematical model.

The harvest data are first used to fit the unknown constants $K_{1}$ and $K_{2}$, while the remaining constants $K_{3}, K_{4}, K_{6}, K_{8}$ are determined using the harvest data or by direct inspection. Their values are found in Table 2 . We vary $K_{1}$ from $4 \times 10^{-8}$ to $1.7 \times 10^{-7}$ with step size $0.5 \times 10^{-8}$ and $K_{2}$ over the range 12.5 to 17.5 with step size 1 . The error between model run and measurements for each combination of $\left(K_{1}, K_{2}\right)$ is calculated. To obtain a realistic initial condition for $D(l, t)$ and $A(l, t)$ we run the model twice for each combination of $\left(K_{1}, K_{2}\right)$. First with an initial condition of $D\left(l, t_{\text {begin }}\right)=10$ stems per square meter and $A\left(l, t_{\text {begin }}\right)=30$ days. Then we use the time averaged values of $A$ and $D$ of the first run as an initial condition for the second run. The final results of the first run are assumed to be a realistic initial condition for the second run. The results of the second run are compared to the measurements as follows

$$
E=\sqrt{\int_{t_{\text {begin }}}^{t_{\text {end }}}(\bar{H}(t)-\tilde{H}(t))^{2} \mathrm{~d} t /\left(t_{\text {begin }}-t_{\text {end }}\right)}
$$

with $\tilde{H}$ the measured harvest rate and $\bar{H}$ the weekly averaged harvest rates from the model output. Note that we use weekly averaged model output since we only have weekly measurements of the harvest rate.

In addition to the model presented in Section 2, we also obtained results for a simplified version of this model. In the simplified model (1a) and (1b) are replaced 
by $D(l, t)=D_{0}$ and $A(l, t)=A_{0}$ with $D_{0}$ and $A_{0}$ constant values representing the average stem density and leaf age over the monitoring period. 


\section{RESULTS}

In this section preliminary predictions of the full model are compared with measurements and with predictions of the simplified version of the full mathematical model. In the first, reference simulation, measurements and simulations of the full model are displayed with an optimal choice of $K_{1}=5.0 \times 10^{-8}$ and $K_{2}=14.5$ based on all harvest data, and the simplified model with the same values for $K_{1}$, see Figure 1a). ( $K_{2}$ is not used in the simplified model since the boundary condition for $D\left(l_{0}, t\right)$ is not used there.) We note that this reference simulation has no predictive value since we fit parameters $K_{1}$ and $K_{2}$ based on the entire harvest period. In a second simulation, $K_{1}=6 \times 10^{-8}$ and $K_{2}=10$ are estimated using only the first half (28 weeks) of the harvest rate data. Subsequently, these values of $K_{1}$ and $K_{2}$ are used to predict the harvest rate. See the results in Figure $1 \mathrm{~b}$ ). We note that this simulation has predictive value beyond week 28 . In Figure 1a) we see that both the full model and the simplified model reproduce the seasonal variability in harvest rates quite well, but larger deviations appear on a weekly scale in both models. When we predict the parameters $K_{1}, K_{2}$ using either the first half of the data set or the entire one, we obtain nearly the same values: $K_{1}=(5.5 \pm 0.5) \times 10^{-8}$ and $K_{2}=12.25 \pm 2.25$. Furthermore, both fits show that the minimum is difficult to determine because it contains shallow local minima. We therefore fixed the parameters by also requiring realistic values for the stem age and stem density functions.

However, the production of the Red Berlin rose is influenced by two distinct events. In weeks 16-26 spider mites were clearly present and injured the leaves. The model calculates a higher production between weeks 23 and 27, but owing to the negative effect of the spider mites on the harvest this is not realized. In weeks 32-37 the uptake of minerals was jeopardized by deviations in the fertigation. We therefore see in weeks 36-40 that the production lags behind the model calculations since the bad mineral uptake has harmed the production. Increased mineral uptake from week 38 onwards allowed a restart of the normal photosynthesis which induced sprouting and a production flush in weeks 44 and 45 . The predictive model of Figure $1 \mathrm{~b}$ shows the production deviation of weeks 36-40 and weeks 44 and 45; since the reference run minimizes all deviations it does not show up that clearly in Figure 1a). The largest deviations of the model can thus be attributed to distinct events unrepresented in the model. The differences between the simplified and full model are not that large, see Figure 1ab), which implies that further improvements are required.

\section{DISCUSSION}

In summary, we presented a mathematical model for rose growth in a greenhouse. Using measured environmental conditions and harvest rates in a greenhouse, we estimated the two most important parameters in the mathematical model via an optimization procedure. The results from the full model and the simplified model are similar (but the latter requires the mean stem density and age values of the former), and some deviations with the harvest occur on a weekly time scale. We 
anticipate that the current work may be improved as follows: I. More advanced optimization techniques for estimating the parameters should be used; in particular a running, weekly update of the parameters based on the last 10 weeks of harvest data and data on the age of the harvested stems (for tuning see also Dayan et al., 2004). II. The expression for the number of sprouting sites, that is, assumption (iv), resulting in the boundary condition for $D\left(l_{0}, t\right)$ requires improvement (e.g. Dayan et al., 1993ab). III. The growth rate velocity could also be extended to include dependencies on nutrient intake and water balance.

ACKNOWLEDGMENTS We thank S. Drost for implementing an early numerical version of the model. O.B. is funded through a fellowship of the Royal Netherlands Academy of Arts and Sciences (KNAW).

\section{Literature Cited}

Bernsen, E., Bokhove, O. and Sar van der Sar, D.M. 2006. Numerical Prediction of Rose Growth. Appendices. Math. Comm. 2006, Department Applied Mathematics, University of Twente, The Netherlands. http://eprints.eemcs.utwente.nl

Bokhove, O., Dubbeldam, J., Getto, P., 't Hof van, B., Ovenden N., Pik, D., Prokert, G., Rottschäfer, V. and Sar van der, D.M. 2002. Roses are unselfish: a greenhouse growth model to predict harvest. Proc. $42^{\text {nd }}$ European study group with industry. Ed.: G.M. Hek. p. 59-76.

Dayan, E. Van Keulen, H. Jones, J.W., Zipori, I, Shmuel, D., and Challa, H. 1993a. Development, calibration and validation of a greenhouse tomato growth model: I. description of the model. Agricultural systems 43:145-163.

Dayan, E. Van Keulen, H. Jones, J.W., Zipori, I, Shmuel, D., Chlla, H. 1993b. Development, calibration and validation of a greenhouse tomato growth model: II. field calibration an validation. Agricultural systems 43: 165-183.

Harley, P.C., Thomas, R.B., Reynolds, J.F., and Strain, B.R. 1992. Modelling photosynthesis of cotton growth in elevated $\mathrm{CO}_{2}$. Plant, Cell, and Environment 15: 271-282.

Dayan, E., Presnov, E., Fuchs, M. 2004. Prediction and calculation of morphological characteristics and distribution of assimilates in the ROSGRO model. Mathematics and Computers in Simulation, 65: 101-116.

Kim, S.-H. and Lieth, J.H. 2001. Modelling diurnal variation of whole-plant photosynthesis of greenhouse roses. Proc. III IS Rose Research, 111-119, 2001.

Kim, S.H. and Lieth J.H. 2003. A coupled model of photosynthesis, stomatal conductance and transpiration for a rose leaf (Rosa hybrida L.) Annals of Botany 91: 771-781.

\section{$\underline{\text { Tables }}$}


Table 1: Model parameters following the notation in Bokhove et al. (2002) for clarity.

\begin{tabular}{lll}
\hline Symbol & Constant & unit \\
\hline$K_{1}$ & mass production per $\mathrm{CO}_{2}$ intake & $\mathrm{kg} / \mu \mathrm{mol}$ \\
$K_{2}$ & birth rate of stems & $\mathrm{s} / \mu \mathrm{mol} / \mathrm{m}$ \\
$K_{3}$ & stem mass per unit length & $\mathrm{kg} / \mathrm{m}$ \\
$K_{4}$ & leaf area of a stem per unit length & $\mathrm{m}$ \\
$K_{6}$ & light absorption coefficient & - \\
$K_{8}$ & leaf area of bush per ground area & - \\
$h$ & height & $\mathrm{m}$ \\
$l$ & length & $\mathrm{m}$ \\
$l_{0}$ & starting length of stems & $\mathrm{m}$ \\
$l_{\text {cut }}$ & cutting length of stems & $\mathrm{m}$ \\
$D$ & stems density distribution & $\mathrm{m}^{-3}$ \\
$A$ & stem age function & $\mathrm{s}$ \\
$v$ & growth velocity & $\mathrm{m} / \mathrm{s}$ \\
$P_{n e t}$ & total net photosynthesis rate & $\mu \mathrm{mol} / \mathrm{m}^{2} / \mathrm{s}$ \\
$H$ & harvest rate & $\mathrm{kg} / \mathrm{m}^{2} / \mathrm{s}$ \\
$M$ & crop mass & $\mathrm{kg} / \mathrm{m}^{2}$ \\
$N$ & number of stems & $\mathrm{m}^{-2}$ \\
$S$ & maximum number of sprouting sites & $\mathrm{m}^{-2}$ \\
$\rho$ & leaf area density & $\mathrm{m}^{-1}$ \\
$a$ & leaf age & $\mathrm{s}$ \\
$I$ & photon flux density & $\mu \mathrm{mol} / \mathrm{m}^{2} / \mathrm{s}$ \\
\hline
\end{tabular}

Table 2: Values of some parameters for the rose variety "Red Berlin".

\begin{tabular}{lll}
\hline Symbol & method & value Red Berlin \\
\hline$K_{1}, K_{2}$ & best fit & - \\
$K_{3}$ & harvest data & $0.047 \mathrm{~kg} / \mathrm{m}$ \\
$K_{4}$ & observation & $0.1 \mathrm{~m}$ \\
$K_{6}$ & from Kim and Lieth (2001) & 0.643 \\
$K_{8}$ & observation & 0.3 \\
$l_{\text {cut }}$ & observation & $l_{0}+0.7 \mathrm{~m}$ \\
$S$ & estimate & $50 \mathrm{~m}^{2} / \mathrm{m}^{2}$ \\
$a_{\text {bush }}$ & estimate & 50 days \\
\hline
\end{tabular}



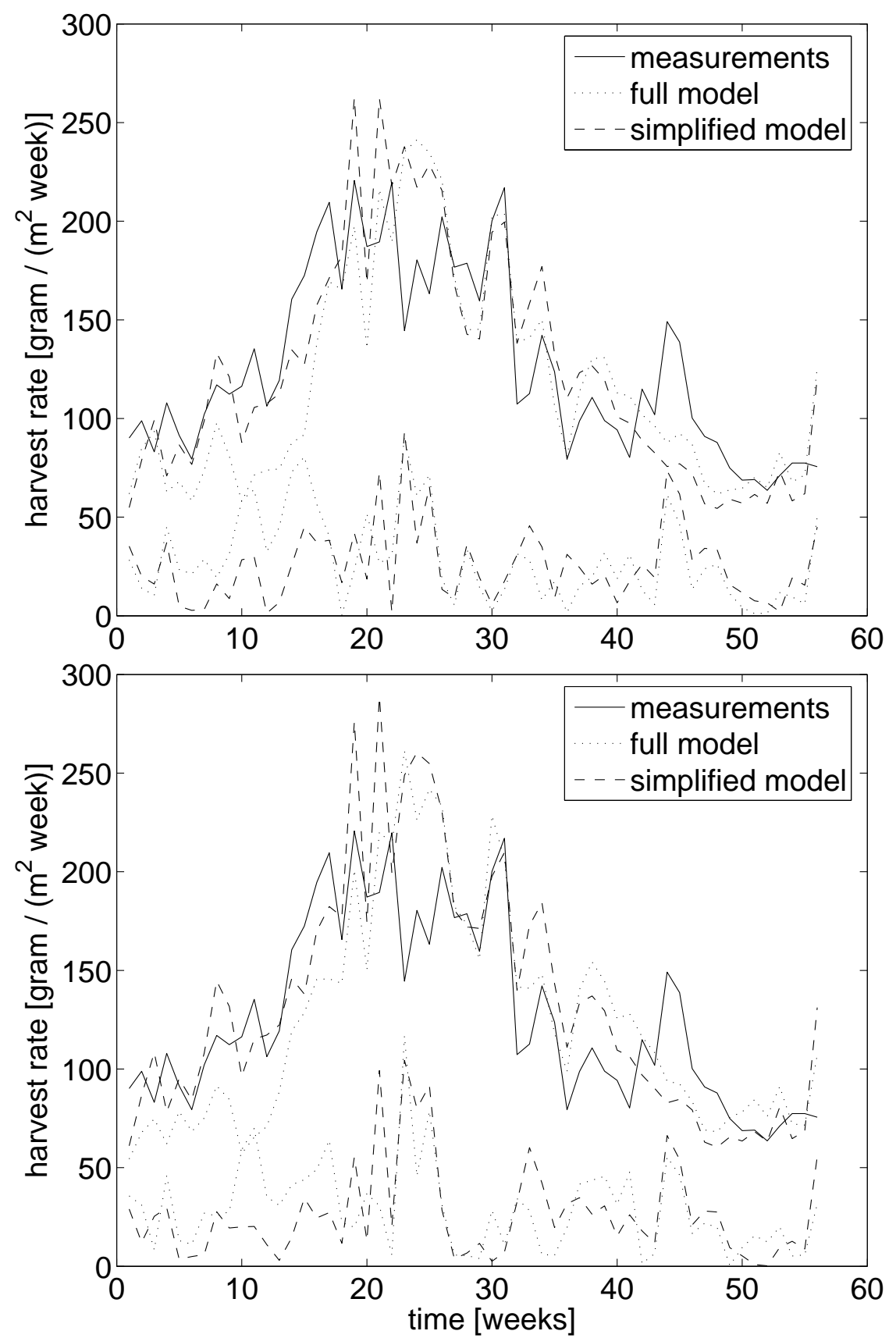

Figure 1: (Top) Weekly averaged harvest rates (fresh weight not dry weight) from the measurements, and the full and simplified model for $K_{1}=5 \times 10^{-8}$ and $K_{2}=$ 14.5, which are fit for the entire period of 55 weeks. Also the absolute value of the error between models and measurements are shown (lower dotted and dashed lines). (Bottom) The same for $K_{1}=6 \times 10^{-8}$ and $K_{2}=10$, but now $K_{1}$ and $K_{2}$ are estimated based on data of the first 28 weeks, and the model is fully predicting the harvest thereafter. 


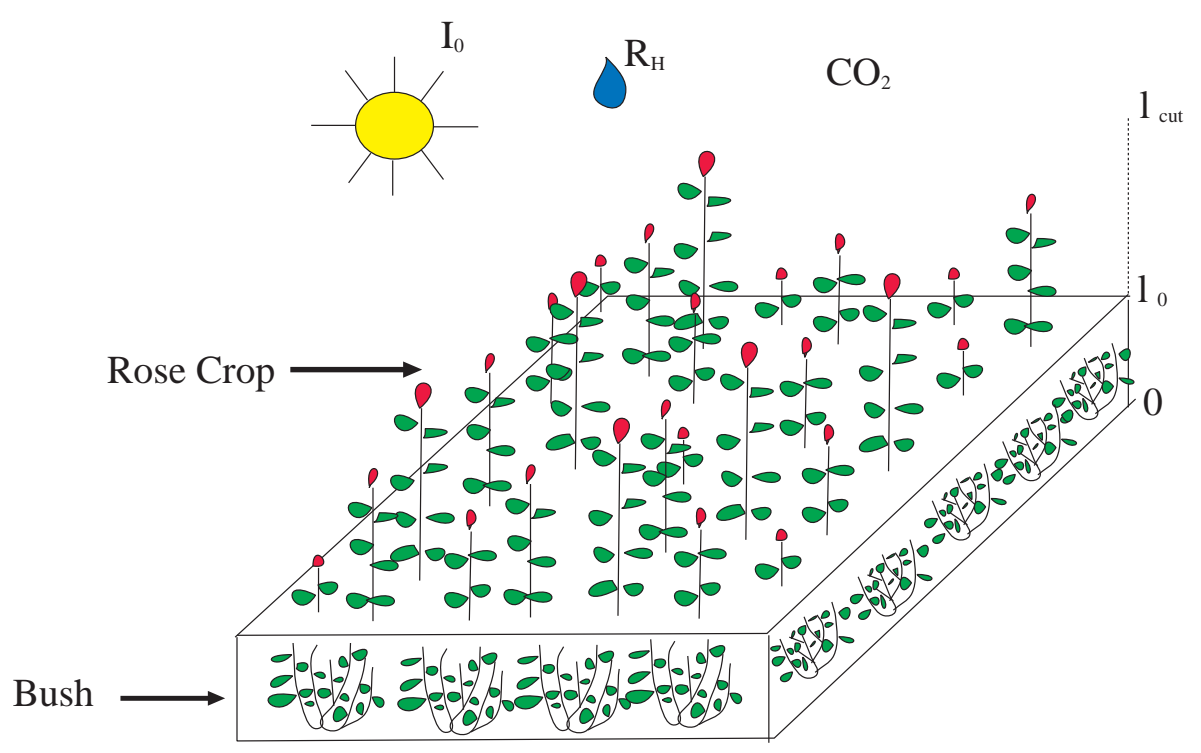

Figure 2: A rose plant is divided into a bush part below $h=l_{0}$, and a crop of rose stems above for $l_{0} \leq h \leq l_{\text {cut }}$, which are harvested when they reach length $l_{\text {cut }}$.

\section{A Derivation of Mathematical Model}

In this section the mathematical model presented before is derived and discussed more thoroughly. For clarity the basic assumptions and definitions in the mathematical model are repeated in this section.

The rose plants growing in a greenhouse can be separated into two parts: the rose stems, which are harvested, and the rose 'bush' below that contains the body of the rose plants supporting each individual stem (see Figure 2). The rose bush is not harvested and lies between heights $0<h<l_{0}$. Bush leaves assimilate energy and contribute to the growth of the crop. At a given time, rose stems growing vertically out of the rose bush have different lengths $l$. As each rose plant consists of a mixture of mature and young rose stems, rose stems of different lengths are distributed throughout the greenhouse.

The mathematical model for rose production is based on the following assumptions, which are based on the experience of one of us - rose growth advisor D.v.d.S. from Phytocare:

i. Roses are unselfish in that all biomass gained through photosynthesis is equally distributed over all stems in a rose plant. In other words, at any fixed time every stem grows with the same speed or growth rate $v(t)$, independent of its own photosynthesis production.

ii. Stems grow vertically.

iii. Stems start growing at height $h=l_{0}$ with adjusted "length" $l=l_{0}$ and are harvested continuously when they reach length $l=l_{\text {cut }}$. Note that lengths are 


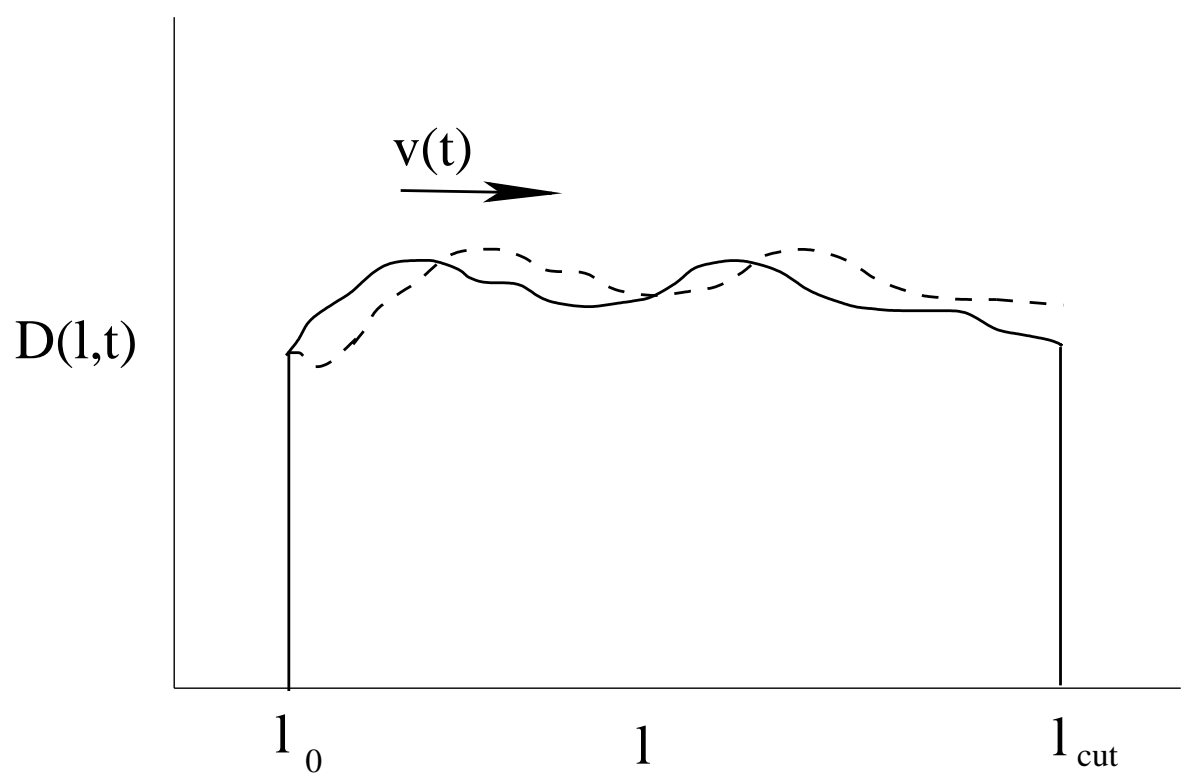

Figure 3: The state of the rose crop can be expressed by a stem density function $D(l, t)$ representing the distribution of stems of differing lengths per square meter of greenhouse area. The dynamics of $D(l, t)$ is governed by an advection equation and the unselfishness principle implies that the advection speed is independent of length $l$.

measured from the bottom of the bush, i.e. from height $h=0$. When a stem is harvested its length is reduced to an adjusted "length" of $l=l_{0}$.

iv. The number of sprouting sites at height $h=l_{0}$ is a continuous, real number when averaged per unit area of greenhouse and proportional to the total photosynthesis but limited by a weighted maximum.

v. New leaves appear at the stem top and are thus the youngest stem leaves. Leaves at height $h=l_{0}$ are therefore the oldest.

vi. Mass and leaf area are proportional to the stem length and uniformly distributed along the stem.

For the dimensions of all occurring quantities and constants we refer to Table 1 and Table 3.

\section{A.1 The stem density function $D$}

The stem density function $D=D(l, t)$ is defined for $l_{0}<l<l_{c u t}$ such that the number of stems of length between $l$ and $l+\mathrm{d} l$ per square meter of greenhouse is $D(l, t) \mathrm{d} l$ (see figure 3). The unselfishness principle stated in assumption (i) implies 


\begin{tabular}{lll}
\hline Symbol & Quantity & unit \\
\hline$h$ & height & $\mathrm{m}$ \\
$l$ & length & $\mathrm{m}$ \\
$l_{0}$ & starting length of stems & $\mathrm{m}$ \\
$l_{\text {cut }}$ & cutting length & $\mathrm{m}$ \\
$D$ & stem density distribution & $\mathrm{m}^{-3}$ \\
$A$ & stem age function & $\mathrm{s}$ \\
$v$ & growth velocity & $\mathrm{m} \mathrm{s}^{-1}$ \\
$P_{n e t}$ & total net photosynthesis rate & $\mu \mathrm{mol} \mathrm{m}^{-2} \mathrm{~s}^{-1}$ \\
$H$ & harvest rate & $\mathrm{kg} \mathrm{m}^{-2} \mathrm{~s}^{-1}$ \\
$M$ & crop mass & $\mathrm{kg} \mathrm{m}^{-2}$ \\
$N$ & number of stems & $\mathrm{m}^{-2}$ \\
$S$ & maximum number of sprouting sites & $\mathrm{m}^{-2}$ \\
$\rho$ & leaf area density & $\mathrm{m}^{-1}$ \\
$q$ & age density distribution & $\mathrm{m}^{-1} \mathrm{~s}^{-1}$ \\
$a$ & leaf age & $\mathrm{s}$ \\
$I$ & photosynthetic photon flux density & $\mu \mathrm{mol} \mathrm{m}^{-2} \mathrm{~s}^{-1}$ \\
$\tau$ & length of growing season (6 months) & $\mathrm{s}$ \\
\hline
\end{tabular}

Table 3: Dimensional quantities used in the global greenhouse model.

that this density function is advected by a growth rate $v=v(t)$, which is independent of $l$ and will be determined later. Hence, we obtain

$$
\partial_{t} D+v \partial_{l} D=0
$$

where $\partial_{t}=\partial / \partial t$ and $\partial_{l}=\partial / \partial l$ denote partial derivatives with respect to time $t$ and length $l$.

The boundary condition at $l=l_{0}$ represents the creation of new stems from the rose bush (provided there is enough light such that $v(t)>0$ ). By assumption (iv), the appearance of new stems at $l_{0}$ is proportional to the rate of photosynthesis $P_{\text {net }}(t)$ and the percentage of free sprouting sites:

$$
D\left(l_{0}, t\right)=K_{2} P_{n e t}(t) \frac{S-N(t)}{S}
$$

with $S$ the maximum number of sprouting sites per square meter of greenhouse and $N(t)$ the total number of stems per square meter given as the integral of the number of stems per unit area $D \mathrm{~d} l$ over the crop from $l_{0}<l<l_{\text {cut }}$ :

$$
N(t)=\int_{l_{0}}^{l_{c u t}} D(l, t) \mathrm{d} l
$$

The net photosynthetic rate $P_{\text {net }}$ in (14) represents the biochemical intake or loss of $\mathrm{CO}_{2}$ per square meter of greenhouse and will be determined later. 


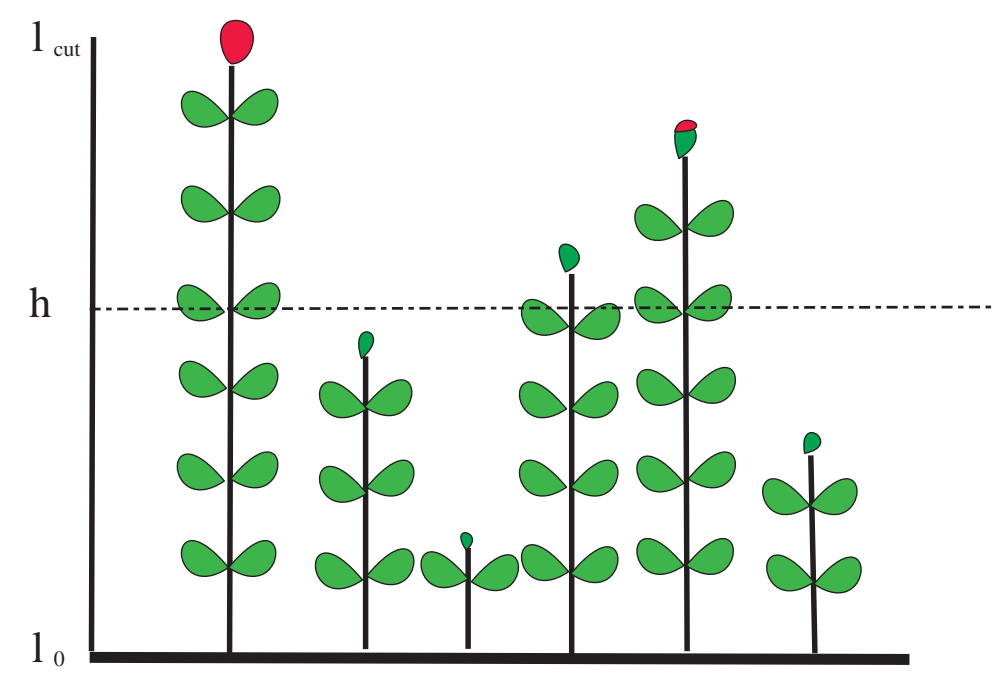

Figure 4: At a certain height $h$ only rose stems of lengths greater than $h$ contribute to the leaf area density $\rho(h)$. Smaller rose stems do not.

Assumption (iii) implies that the harvest rate $H(t)$ per square meter of greenhouse is given by

$$
H(t)=K_{3} v(t)\left(l_{c u t}-l_{0}\right) v(t)
$$

where $K_{3}$ is the mass of a rose per unit length.

\section{A.2 Determining the growth speed $v$}

The mass of crop in the greenhouse is again proportional to $K_{3}$ and the first moment in $l$ of the stem density function

$$
M(t)=K_{3} \int_{l_{0}}^{l_{c u t}}\left(l-l_{0}\right) D(l, t) \mathrm{d} l,
$$

where the stem density is properly weighed by the stem length in the crop $\left(l-l_{0}\right)$ in order to obtain the mass, following assumption (vi). This first moment accounts for a longer stem carrying more mass in proportion to its length than a shorter stem. Differentiating (17), integrating the r.h.s. by parts, and using (16) gives

$$
\begin{aligned}
\frac{\mathrm{d} M}{\mathrm{~d} t} & =-K_{3} v(t) \int_{l_{0}}^{l_{c u t}}\left(l-l_{0}\right) \partial_{l} D(l, t) \mathrm{d} l \\
& =K_{3} v(t) \int_{l_{0}}^{l_{c u t}} D(l, t) \mathrm{d} l-K_{3} v(t)\left(l_{\text {cut }}-l_{0}\right) D\left(l_{\text {cut }}, t\right) \\
& =K_{3} v(t) N(t)-H(t) .
\end{aligned}
$$


The net photosynthetic rate $P_{n e t}$ is proportional to the change in productive mass plus the harvest rate, and by using (18) we can subsequently obtain the growth rate $v(t)$ :

$$
K_{1} P_{n e t}(t)=\frac{\mathrm{d} M}{\mathrm{~d} t}+H(t)=K_{3} v(t) N(t) \quad \Longleftrightarrow \quad v(t)=\frac{K_{1} P_{n e t}(t)}{K_{3} N(t)},
$$

where $K_{1}$ is the mass production per $\mathrm{CO}_{2}$ intake.

\section{A.3 The stem age function $A$}

Assumption (i) implies that all stems grow with the same rate of growth $v$. The age of a stem therefore follows directly from its length. The stem age function $A=A(l, t)$ is defined implicitly by the following relation

$$
\int_{t-A(l, t)}^{t} v\left(t^{\prime}\right) \mathrm{d} t^{\prime}=l-l_{0}
$$

Differentiating (20) with respect to $l$ and $t$ results in

$$
v(t-A(l, t)) \partial_{l} A(l, t)=1
$$

and

$$
v(t)-v(t-A(l, t))\left(1-\partial_{t} A(l, t)\right)=0,
$$

respectively. Combining (21) and (22) gives the following partial differential equation for $A$

$$
\partial_{t} A+v \partial_{l} A=1
$$

The boundary conditions for $A$ at $l=l_{0}$ follows directly from (20)

$$
A\left(l_{0}, t\right)=0
$$

\section{A.4 The leaf density functions}

In order to calculate $P_{n e t}$ from the local photosynthesis model, we require information about the distribution of leaf area, leaf age and light intensity (photon flux density) with respect to the height $h$ of a leaf on a stem.

The leaf density function $\rho(h, t)$ is defined so that $\rho(h, t) \mathrm{d} h$ yields the area of leaves at height between $h$ and $h+\mathrm{d} h$ per square meter of greenhouse. It is related to $D(h, t)$ by

$$
\rho(h, t)=K_{4} \int_{h}^{l_{c u t}} D\left(l^{\prime}, t\right) \mathrm{d} l^{\prime}
$$

with $K_{4}$ the leaf area of a stem per unit length. The integration limits are chosen as $h$ and $l_{\text {cut }}$ because only rose stems with lengths greater than $h$ contribute to the leaf area density at the height $h$; a graphical explanation is provided in Figure 4. 
The age density distribution $q(h, a, t)$ is defined so that $q(h, a, t) \mathrm{d} h \mathrm{~d} a$ yields the leaf area of age between $a$ and $a+\mathrm{d} a$ located between the heights $h$ and $h+\mathrm{d} h$ per square meter of greenhouse.

To find an expression for the age density function we first note that the leaf density function can be expressed in the age density function as follows

$$
\rho(h, t)=\int_{0}^{A\left(l_{0}+l_{c u t}-h, t\right)} q\left(h, a^{\prime}, t\right) \mathrm{d} a^{\prime},
$$

where the upper and lower integration limits are choosen as the maximum and minimum age, respectively, of a leaf at height $h$ or higher. Consider the case $h=l_{0}$, then the upper limit is the maximum age of the density distribution function concerning stems with lengths between $l_{0}<l<l_{c u t}$, which is the age $A\left(l_{c u t}, t\right)$ of a stem of length $l_{\text {cut }}$ indeed. Consider the case $h=l_{\text {cut }}$, then the upper limit is the maximum age of the density distribution function concerning stems with lengths between $l_{\text {cut }}<l<l_{\text {cut }}$, which is the age $A\left(l_{0}, t\right)=0$ of a stem of length $l_{0}$. By virtue of the unselfishness principle, the age $a$ of a leaf at height $h$ is the same as the age $A\left(l_{0}+l_{c u t}-h\right)$ of a stem of length $l_{0}+l_{c u t}-h$. This is shown graphically in Figure 5a), where the age of top part $l_{c u t}-h$ of the stem with length $l_{c u t}$ between $h$ and $l_{\text {cut }}$ is the same as the age of the stem of length $l_{0}+l_{c u t}-h$ because all stems grow with the same speed $v(t)$ in time. In this expression $a^{\prime}$ is the age of a leaf at height $h$ on a stem of length $l$. We introduce the coordinate transformation $A\left(l_{0}+l^{\prime}-h, t\right)=a^{\prime}$, explained in Figure $\left.5 \mathrm{~b}\right)$, such that $l^{\prime}$ is the length of a stem which has leaves of age $a^{\prime}$ at height $h$. We now have

$$
\mathrm{d} a^{\prime}=\partial_{l^{\prime}} A\left(l_{0}+l^{\prime}-h, t\right)=\frac{\mathrm{d} l^{\prime}}{v\left(t-A\left(l_{0}+l^{\prime}-h, t\right)\right)},
$$

where $\partial_{l^{\prime}} A\left(l_{0}+l^{\prime}-h, t\right)$ follows from differentiation to $l$ of (20). Hence we have

$$
\rho(h, t)=\int_{h}^{l_{\text {cut }}} \frac{q\left(h, A\left(l_{0}+l^{\prime}-h, t\right), t\right)}{v\left(t-A\left(l_{0}+l^{\prime}-h, t\right)\right)} \mathrm{d} l^{\prime} .
$$

Since (27) and (24) should hold for all $l_{0} \leq h \leq l_{\text {cut }}$ we must have that

$$
q(h, a, t)=K_{4} v(t-a) D\left(h+\int_{t-a}^{t} v\left(t^{\prime}\right) \mathrm{d} t^{\prime}, t\right) .
$$

\section{A.5 The photosynthesis in the crop}

The top leaves of the tallest rose stems receive all the light available. However, the amount of light reaching the lower leaves of mature plants and of newer stems is diminished by the amount of leaf coverage above. The isotropic nature of the greenhouse means that all leaves at the same height have approximately the same 
a)

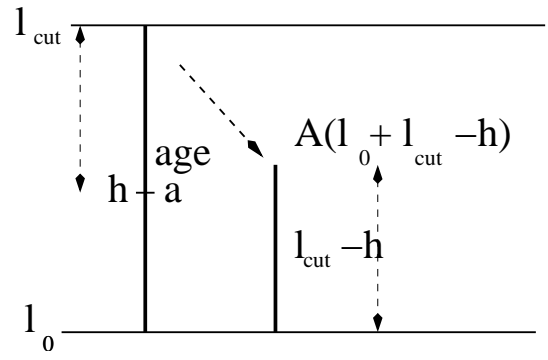

b)

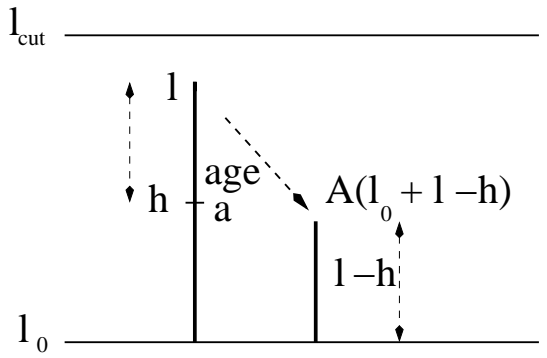

Figure 5: (a) The age of a leaf at height $h$ on a stem with a top part $l_{\text {cut }}-h$ lying between $h$ and $l_{c u t}$ of the stem with length $l_{c u t}$ is the same as the age of the stem of length $l_{0}+l_{\text {cut }}-h$ because all stems grow with the same speed $v(t)$ in time. (b) The age of a leaf at height $h$ on a stem with a top part $l-h$ lying between $h$ and $l$ of the stem with length $l$ is the same as the age of the stem of length $l_{0}+l-h$ because all stems grow with the same speed.

amount of shade. The change in light intensity $I(h)$ as function of $h$ is thus taken to be proportional to the area of leaves at height $h$, that is $\rho(h) \mathrm{d} h$, and $I(h)$, leading to

$$
\frac{d I(h)}{d h}=K_{6} \rho(h) I(h), \quad I\left(l_{\text {cut }}\right)=I_{0} \quad \Longleftrightarrow \quad I(h)=I_{0} e^{-K_{6} \int_{h}^{l_{c u t}} \rho(\zeta) \mathrm{d} \zeta}
$$

with $K_{6}$ the dimensionless light absorption coefficient, given in Kim and Lieth (2001) as 0.643 (their expression (10)). Note that $\int_{h}^{l_{c u t}} \rho(\zeta) \mathrm{d} \zeta$ is the cumulative leaf area index from the top.

The assumed age distribution of the leaves of the rose stems and the change in light intensity at each height now enable us to calculate the net photosynthesis produced by the rose crop per square meter of greenhouse, as follows

$$
\begin{gathered}
P_{\text {crop }}(t)=\int_{l_{0}}^{l_{\text {cut }}} \int_{0}^{A\left(l_{0}+l_{\text {cut }}-h^{\prime}, t\right)} q\left(h^{\prime}, a^{\prime}, t\right) P_{\text {leaf }}\left(a^{\prime}, I\left(h^{\prime}\right), t\right) \mathrm{d} a^{\prime} \mathrm{d} h^{\prime} \\
=K_{4} \int_{l_{0}}^{l_{\text {cut }}} \int_{0}^{A\left(l_{0}+l_{\text {cut }}-h^{\prime}, t\right)} v\left(t-a^{\prime}\right) D\left(h^{\prime}+\int_{t-a^{\prime}}^{t} v\left(t^{\prime}\right) \mathrm{d} t^{\prime}, t\right) \times \\
P_{\text {leaf }}\left(a^{\prime}, I\left(h^{\prime}\right), t\right) \mathrm{d} a^{\prime} \mathrm{d} h^{\prime} .
\end{gathered}
$$

Here $P_{\text {leaf }}(a, I(h), t)$ is the local photosynthesis rate per unit area for a leaf receiving light of an intensity $I(h)$ at height $h$ and of age $a$ under given exterior climatic conditions (that is, temperature, relative air humidity, light intensity, and $\mathrm{CO}_{2}$-concentration) at time $t$, as predicted by the local leaf model described in Appendix A.7. Using the coordinate transformation $a^{\prime}=A\left(l_{0}+l^{\prime}-h^{\prime}, t\right)$ explained in Figure 5b), expression (30) reduces to

$$
P_{\text {crop }}(t)=K_{4} \int_{l_{0}}^{l_{\text {cut }}} \int_{h^{\prime}}^{l_{\text {cut }}} D\left(l^{\prime}, t\right) P_{\text {leaf }}\left(A\left(l_{0}+l^{\prime}-h^{\prime}, t\right), I\left(h^{\prime}\right), t\right) \mathrm{d} l^{\prime} \mathrm{d} h^{\prime} .
$$


One more coordinate transformation $l=l_{0}+l^{\prime}-h^{\prime}$ reduces (31) to (7) in the main text

$$
P_{\text {crop }}(t)=K_{4} \int_{l_{0}}^{l_{\text {cut }}} \int_{l_{0}}^{l_{0}+l_{\text {cut }}-h} D\left(l-l_{0}+h, t\right) P_{\text {leaf }}(A(l, t), I(h, t), t) \mathrm{d} l \mathrm{~d} h .
$$

\section{A.6 The photosynthesis in the bush}

While $P_{\text {crop }}$ represents the major source of biomass for roses in the greenhouse, the rose bush below $l_{0}$ also contains leaves and produces an additional seasonallyvarying contribution to the net growth rate. We assume that the leaves in the bush all have a mean age $a_{\text {bush }}=\tau / 2$ and light of mean intensity $I\left(l_{0}\right)$, where $\tau$ is the length of an entire growing season. Introducing another constant $K_{8}$ to represent the leaf area in the bush, we obtain

$$
P_{\text {bush }}=K_{8} P_{\text {leaf }}\left(a_{\text {bush }}, I\left(l_{0}\right), t\right)
$$

for the net rate of photosynthesis of the bush.

Finally, the total net photosynthetic rate is the sum of the net crop photosynthesis from (31) and the net bush photosynthesis from (33) leading to

$$
P_{\text {net }}(t)=P_{\text {crop }}(t)+P_{\text {bush }}(t)
$$

\section{A.7 Local leaf model for photosynthesis}

It is important to emphasize that the production model described in the previous section is closed once only we have a model for the local photosynthesis $P_{\text {leaf }}(a, I, t)$ in a leaf. Presently, we will use a version of the photosynthesis rose leaf model of Harley et al. (1992) and Kim and Lieth (2001).

Following Harley et al. (1992) and Kim and Lieth (2001), the photosynthetic rate in a unit area of leaf receiving light of intensity $I$ and with age $a$, is given by

$$
P_{\text {leaf }}(a, I, t)=\min \left\{A_{v}, A_{j}\right\}-R_{d}
$$

Here $A_{v}$ and $A_{j}$ are the rate of Rubisco limited photosynthesis and the rate limited by RuBP regeneration, respectively, while $R_{d}$ is a threshold $\mathrm{CO}_{2}$ consumption or dark respiration, for example due to losses at night, which we take constant at $R_{d}=$ $0.82 \mu \mathrm{mol} /\left(\mathrm{m}^{2} \mathrm{~s}\right)$ (cf. Hartley et al., 1992). The existence of the dark respiration term $R_{d}$ implies that $P_{\text {leaf }}(a, I, t)$ can be less than zero. However, any local losses can be compensated by a positive global photosynthesis rate elsewhere due to the unselfishness principle. Both $P_{\text {net }}$ and $P_{\text {leaf }}(a, I, t)$ are expressed in terms of a $\mathrm{CO}_{2}$ rate per square meter of greenhouse, which is $\mu \mathrm{mol} \mathrm{CO}_{2} /\left(\mathrm{m}^{2} \mathrm{~s}\right)$.

The photosynthesis rates $A_{v}$ and $A_{j}$ depend on the intercellular $\mathrm{CO}_{2}$-concentration $C_{i}$, as is shown in figure 6: the photosynthesis stops in conditions of too little intercellular $\mathrm{CO}_{2}$ (i.e. if $C_{i}<\Gamma_{*}$ ). For increasing values of $C_{i}$, the photosynthetic rate 


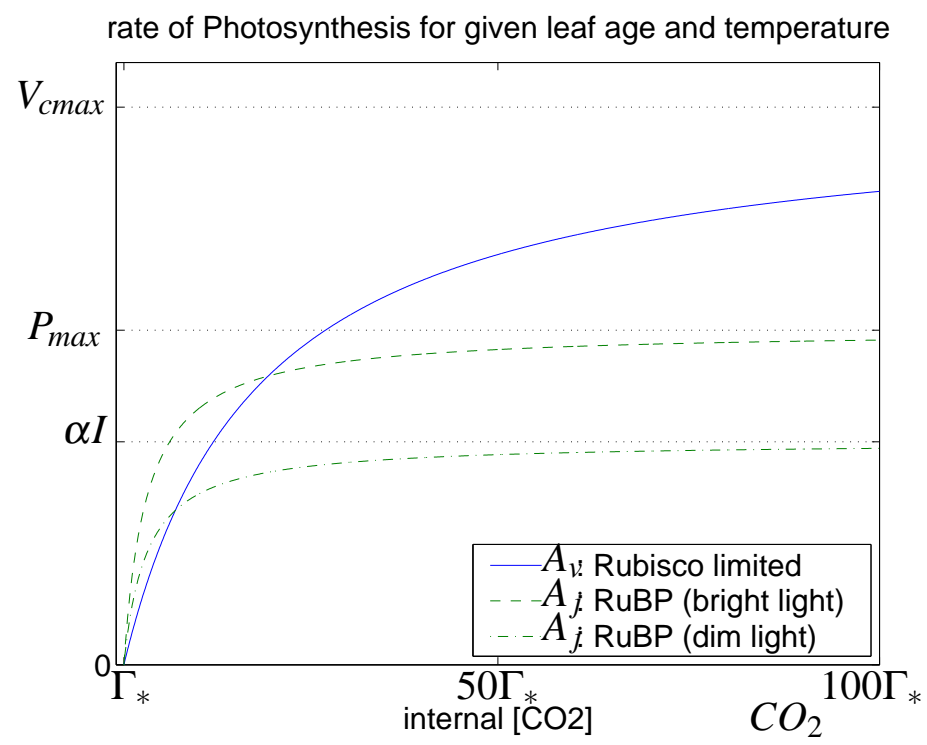

Figure 6: The $C_{i}$-dependence of photosynthesis rates $A_{v}$ and $A_{j}$.

allowed by RuBP regeneration increases faster than the Rubisco limited photosynthesis rate, but the latter attains a higher value $V_{c \max }$ than the former.

The formula for Rubisco limited photosynthesis $A_{v}$ is given by (Kim and Lieth, 2001)

$$
A_{v}=V_{c \max } \frac{C_{i}-\Gamma_{*}}{C_{i}+\kappa} \quad \text { with } \quad V_{c \max }=V_{m} g(T) f(a),
$$

where $V_{c \max }$ is the maximum rate of carboxylation and $C_{i}$ the intercellular $\mathrm{CO}_{2}$ concentration, and $g(T)$ and $f(a)$ represent the dependence on leaf temperature $T$ and leaf age $a$. The remaining unknowns are constants, defined and given in table 4; see also Harley et al. (1992) and Kim and Lieth (2001). The RuBP limited photosynthetic rate $A_{j}$ is

$$
A_{j}=\frac{C_{i}-\Gamma_{*}}{4\left(C_{i}+2 \Gamma_{*}\right)} J
$$

with the potential electron transport rate $J$ given by

$$
J=\frac{8 \alpha I P_{\max }}{\alpha I+P_{\max }+\sqrt{\left(\alpha I+P_{\max }\right)^{2}-4 \alpha I P_{\max } \theta}}
$$

and

$$
P_{\text {max }}=P_{m} g(T) f(a),
$$

where $I=I(h)$ is the photosynthetic flux density given in (29) at height $h$ above the ground. We note that when $P_{\max } \gg \alpha I$ the potential rate $J \approx 4 \alpha I$, which 


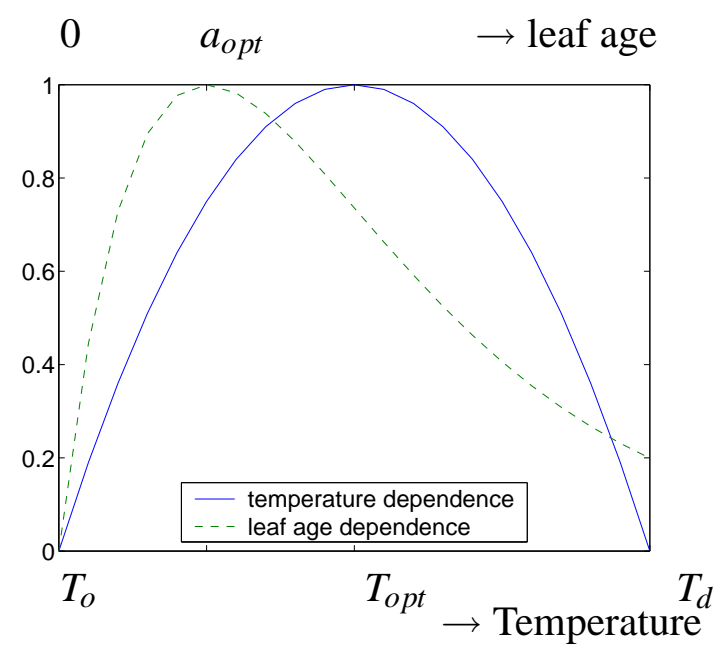

Figure 7: The temperature and leaf age dependence of local photosynthetic rates $V_{\text {cmax }}$ and $P_{\text {max }}$.

means that when $\alpha I$ is sufficiently small the production is limited by the lack of light. Inversely, when $P_{\max } \ll \alpha I$ the potential rate $J \approx 4 P_{\max }$, which means that when $\alpha I$ is sufficiently large, any increase in the amount of light has no additional influence on the rate of photosynthesis.

The temperature dependence $g(T)$ and age dependence $f(a)$ of the photosynthesis rates $V_{\text {cmax }}$ and $P_{\max }$ are shown in figure 7 . These dependencies are described by the formula

$$
g(T)=\frac{4\left(T-T_{o}\right)\left(T_{d}-T\right)}{\left(T_{d}-T_{o}\right)^{2}} \quad \text { and } \quad f(a)=\left(a / a_{o p t}\right) e^{\left(1-a / a_{o p t}\right)} .
$$

This temperature dependence $g(T)$ is chosen, instead of the one in Harley et al. (1992) and Kim and Lieth (2001), because it includes a minimum and maximum temperature $T_{o}$ and $T_{d}$, respectively, below and above which the photosynthesis production is zero, respectively. The age dependence $f(a)$ follows from Lieth and Pasian (1990).

The intercellular $\mathrm{CO}_{2}$-concentration is

$$
C_{i}=C_{a}-\beta \frac{P_{\text {leaf }}(a, I, t)}{g_{s}} \quad \text { with } \quad g_{s}=g_{0}+g_{1} P_{\text {leaf }}(a, I, t) R_{H} / C_{a},
$$

where the ambient $\mathrm{CO}_{2}$-concentration is given in $\mu \mathrm{mol} \mathrm{CO}_{2} /\left(\mathrm{mol}\right.$ air), $R_{\mathrm{H}}$ is the relative humidity, $g_{s}$ is the stomatal conductance to $\mathrm{H}_{2} \mathrm{O}$ in $\mathrm{mol} \mathrm{H}_{2} \mathrm{O} /\left(\mathrm{m}^{2} \mathrm{~s}\right), g_{0}$ is the minimal stomatal conductance to $\mathrm{H}_{2} \mathrm{O}$ in $\mathrm{mol} \mathrm{H}_{2} \mathrm{O} /\left(\mathrm{m}^{2} \mathrm{~s}\right)$, and $\beta=1.6$. Note 


\begin{tabular}{|c|c|c|}
\hline Constant & definition & value \\
\hline$R_{d}$ & dark respiration & $0.82 \mu \mathrm{mol} \mathrm{CO} 2 /\left(\mathrm{m}^{2} \mathrm{~s}\right)$ \\
\hline$V_{m}$ & - & $94.3 \mu \mathrm{mol} \mathrm{CO}_{2} /\left(\mathrm{m}^{2} \mathrm{~s}\right)$ \\
\hline$\Gamma_{*}$ & $\mathrm{CO}_{2}$ compensation point & $44 \mu \mathrm{mol} C O_{2} / \mathrm{mol}$ \\
\hline$\kappa$ & - & $730 \mu \mathrm{mol} \mathrm{CO}_{2} / \mathrm{mol}$ \\
\hline$P_{m}$ & - & $56.6 \mu \mathrm{mol} \mathrm{CO} /\left(\mathrm{m}^{2} \mathrm{~s}\right)$ \\
\hline$\alpha$ & quantum efficiency & $0.055 \mathrm{~mol} \mathrm{CO}_{2} /(\mathrm{mol}$ photon $)$ \\
\hline$\theta$ & curvature factor & 0.7 \\
\hline$T_{o}$ & lower temperature bound & $10^{\circ} \mathrm{C}$ \\
\hline$T_{d}$ & upper temperature bound & $48.6^{\circ} \mathrm{C}$ \\
\hline$a_{\text {opt }}$ & optimum age & 28.01 days \\
\hline$g_{0}$ & minimum stomatal conductance & $0.18 \mathrm{~mol} \mathrm{H}_{2} \mathrm{O} /\left(\mathrm{m}^{2} \mathrm{~s}\right)$ \\
\hline$g_{1}$ & - & 6.71 \\
\hline$\beta$ & conversion factor $\mathrm{CO}_{2} / \mathrm{H}_{2} \mathrm{O}$ & 1.6 \\
\hline
\end{tabular}

Table 4: Definitions and given values of the constants used in the leaf photosynthesis model.

that (41) is the quasi steady-state solution of

$$
\frac{\partial C_{i}}{\partial t}=g_{s}\left(C_{a}-C_{i}\right)-\beta P(t, a, h)
$$

expressing the effects of consumption of $\mathrm{CO}_{2}$ by photosynthesis and conduction of $\mathrm{CO}_{2}$ by the leaf stomata. Note also that the intercellular concentration $C_{i}$ is lower than the ambient one, provided $P_{\text {leaf }}(a, I, t)>0$. For an increasing production $P_{\text {leaf }}(a, I, t)$ the concentration $C_{i}$ is decreasing, while for increasing ambient humidity $R_{H}$ the concentration $C_{i}$ is increasing.

The leaf temperature $T$ and the ambient temperature $T_{a}$ are related by linearizing the expression in Jones (1992; page 232)

$$
T=T_{a}+\left[4-(2 / 45) T_{a}\right] I(h) /(1380)-\left[1+(6 / 45) T_{a}\right]\left(1-R_{H}\right) / 0.7
$$

with $I(h)$ expressed as $\mu$ mol photons $/\left(\mathrm{m}^{2} \mathrm{~s}\right)$ and $R_{H}$ taking some value between 0 and 1.

The calculation of the photosynthesis rate $P(t, a, h)$ requires the solution of a quadratic equation, since the intercellular $\mathrm{CO}_{2}$-concentration $C_{i}$ and the stomatal conductance $g_{s}$ both depend on $P(t, a, h)$.

\section{B Numerical Method}

In this section we discuss the numerical method used to solve the model equations. We discretize the advection equations for $A$ and $D$ using a finite volume method 
using an upwind flux. Assume that the interval $\left[l_{0}, l_{\text {cut }}\right]$ is discretized into $N$ intervals. Then we have $l_{0}<l_{1}<l_{2}<\ldots<l_{N}=l_{\text {cut }}$. On each interval $\left[l_{i-1}, l_{i}\right]$ for $i=1,2, \ldots, N$, we approximate the exact solution of $D$ and $A$ with a mean value $D_{i}$ and $A_{i}$. Substituting this numerical approximation in (1a) integrating over one interval $\left[l_{i-1}, l_{i}\right]$ results in

$$
\begin{aligned}
0 & =\int_{l_{i-1}}^{l_{i}} \partial_{t} D \mathrm{~d} l+v(t) \int_{l_{i-1}}^{l_{i}} \partial_{l} D \mathrm{~d} l \\
& =\Delta l_{i} \frac{\mathrm{d} D_{i}}{\mathrm{~d} t}+v(t)\left[D\left(l_{i}, t\right)-D\left(l_{i-1}, t\right)\right]
\end{aligned}
$$

with $\Delta l_{i}=l_{i}-l_{i-1}$. In the last term of this equation there is a problem since the numerical approximation is not well defined for $l=l_{i}$ (and $l=l_{i+1}$ ). We replace the value of $D$ at these points by an upwind flux, which is defined by

$$
\hat{D}_{i}=\left\{\begin{array}{ll}
D_{i-1} & \text { if } v(t) \geq 0 \\
D_{i} & \text { if } v(t)<0
\end{array} .\right.
$$

We then have (assuming that $v(t)>0$ )

$$
0=\Delta l_{i} \frac{\mathrm{d} D_{i}}{\mathrm{~d} t}+v(t)\left(\hat{D}_{i}-\hat{D}_{i-1}\right) .
$$

We use a forward Euler scheme for the time discretization of $D_{i}$ to obtain

$$
D_{i}(t+\Delta t)=D_{i}(t)-\frac{v(t) \Delta t}{\Delta l_{i}}\left(\hat{D}_{i}(t)-\hat{D}(t)_{i-1}\right) .
$$

Discretizing (1b) with a similar approach results in

$$
A_{i}(t+\Delta t)=A_{i}(t)-\frac{v(t) \Delta t}{\Delta l_{i}}\left(\hat{A}(t)_{i}-\hat{A}(t)_{i-1}\right)+\Delta t .
$$

The size of the time step, $\Delta t$, is limited by the so called CFL condition:

$$
\Delta t \leq \min _{i=1,2, \ldots, N} \Delta l_{i} / v(t)
$$

This condition ensures that the numerical solution is stable.

For the numerical approximation of $v(t)$ we use the following Riemann sums to 
approximate the integrals appearing in (15), (24), (29), (32) and (33):

$$
\begin{aligned}
& N(t) \approx N_{i}=\sum_{i=1}^{N} \Delta l_{i} D_{i} \\
& \rho(h, t) \approx \rho_{i}=K_{4}\left(-\Delta l_{i} D_{i} / 2+\sum_{j=i}^{N} \Delta l_{j} D_{j}\right) \\
& I(h, t) \approx I_{i}=I_{0} \exp \left(K_{6}\left(\Delta l_{i} \rho_{i} / 2-\sum_{j=i}^{N} \Delta l_{j} \rho_{j}\right)\right) \\
& P_{\text {crop }}(t) \approx K_{4} \sum_{i=1}^{N} \sum_{j=1}^{N-i+1} D_{i+j} P_{\text {leaf }}\left(A_{j}, I_{i}, t\right) \Delta l_{i} \Delta l_{j} \\
& P_{\text {bush }}(t) \approx K_{8} P_{\text {leaf }}\left(a_{\text {bush }}, I_{1}, t\right) .
\end{aligned}
$$

For the calculation of $P_{l e a f}$ we need the climate conditions in the greenhouse $T_{a}$, $R_{H}, C_{a}$ and $I_{0}$. These values are given only at discrete times and hence we use linear interpolation to obtain these variables at any point in time.

\section{Implementation}

The numerical scheme is implemented in MATLAB using the assumption that the sizes of all intervals $\left[l_{i-1}, l_{i}\right]$ are equal, i.e. $\Delta l=\left[l_{i-1}, l_{i}\right]$. Here we give only a short overview of the code. The following MATLAB functions are used to implement the model: P_leaf, P_total and grow. Additionally the script constants.m defines some constants used throughout the whole program.

The $\mathrm{P}=\mathrm{P}$ _leaf ( $\mathrm{a}, \mathrm{I}, \mathrm{climate}$, params) function computes the local photosynthesis rate for the leafs. Using a vector a of leaf ages and a vector I of light intensities the photosynthesis in a single leaf is computed. Note that this function is vectorized, i.e. it accepts vectors of ages and light intensities and returns the matrix $\mathrm{P}$ containing the photosynthesis rates for all combinations of age and light intensity. The params argument in this function is an array containing the values of the model parameters $\left(K_{1}, K, K_{3}, K_{4}, K_{5}, K_{8}, R_{d}, \alpha, \beta, \kappa, \theta, \Gamma_{*}, P_{m}, V_{m}, g_{0}, g_{1}, T_{0}\right.$, $T_{D}$ and $\left.a_{\text {opt }}\right)$. In the file constants.m the parameters which are represented by the elements of this array can be found. For instance params (iK1) should store the value of $K_{1}$. In a similar way the climate variables $\left(T_{a}, C_{a}, R_{h}\right.$ and $\left.I_{0}\right)$ are stored in the climate array.

The P=P_total (dh, d, A, climate, params) function calculates the sum of the photosynthesis of all leafs. The climate data and parameter values are given again by the array climate and params. Further this function requires the element size $\mathrm{dh}$ and the mean values of $A$ and $D$ on each interval stored in array $\mathrm{d}$ and $\mathrm{A}$.

Finally, the function $[d, A, v, H, P, N, d t]=\operatorname{grow}(d h, d 0, A 0$, max_dt, t_output, t_climate_data, cliamte_data, params) implements the time stepping scheme. This function requires the following arguments: The element size dh, 
the initial conditions for $D$ and $A$ given by d0 and A0, the maximum size of a time step dt, an array t_output containing the times at which output data should be given, the climate data at all times given by t_climate_data and climate_data, and finally the values of the model parameters given by params. This function calculates how $D$ and $A$ evolve over the time interval from min(t_output) to $\max$ (t_output). The size of the time step is at most equal to max_dt but can be smaller if required by the CFL condition, for instance. The output variables of this function are average values (averaged over the time intervals following from the t_output variable) of the functions $D$ and $A$, the growth rate $v$, the harvest rate $H$, the photosynthesis rate $P_{n e t}$, the total number of stems per square metre of greenhouse $N$ and the average value of the time step taken. The matrices $\mathrm{d}$ and A are used to store in each column the time averaged mean values of $D$ and $A$ on each interval $\left[h_{i-1}, h_{i}\right]$. The vectors $\mathrm{v}, \mathrm{H}, \mathrm{P}$ and $\mathrm{N}$ are used to store the time averaged values of the corresponding variables.

In addition to these three most important functions there is a function optimize which is a very primitive optimization routine for fitting the parameters. There are functions read_climate_data and read_harvest_data to read the climate data and harvest data from a file.

Finally there are the scripts init.m, default_parameters.m simulate.m and fit.m. These script should make it easier to call the functions optimize and grow.

\section{Details of Simulations}

Here we give some details of the simulations discussed in the results section. The number of elements used in the numerical method was $N=50$ and the timestep was determined by the following expression

$$
\Delta t=\min \left(\Delta t_{\max }, C F L \min _{i=1,2, \ldots, N} \Delta l / v_{i}\right)
$$

with the maximum timestep size $\Delta t_{\max }=4$ hours and $C F L=0.9$. Further we took $l_{\text {cut }}-l_{0}=0.8 \mathrm{~m}$.

The environmental conditions of Red Berlin rose variety in the period January 1st 2001 through till January 25th 2002 are given in Figure 11.

The reference simulation uses th entire harvest period of 56 weeks to fit the parameters $K_{1}$ and $K_{2}$, while the predictive simulation uses the first 28 weeks of harvest to fit $K_{1}$ and $K_{2}$, whereafter it uses the fitted values to predict the harvest given the ambient environmental conditions as function of time. Model harvest rates are displayed in Figure 1a,b) in the main text. It turns out that $K_{2}$ is difficult to optimize it barely varies as function of $K_{2}$. The $K_{1}, K_{2}$-landscape consists of a valley with local and global minima, see Figure 10, Figure 8, and Figure 9. This is not surprising since by crudely averaging the relations (2), (3), (4), and (11) one 
finds

$$
K_{2}=K_{1} \frac{\bar{D}\left(l_{0}\right) \bar{D}\left(l_{c u t}\right)\left(l_{c u t}-l_{0}\right) S}{\bar{H}(S-\bar{N})}
$$

with averages $\bar{H}, \bar{N}, \bar{D}\left(l_{0}\right), \bar{D}\left(l_{\text {cut }}\right)$. We chose the simulation for which the stem and age density distribution appeared most realistic. Implicitly, a second visual optimization was therefore used: results as in Figure 14 were thus rejected.

The selected stem age distribution as function of time and stem length is given in Figure 12 for both simulations in the main text. The stem density function as function of time and stem length is displayed in Figure 13. The high production in Figure 1 at the end of week 56 is caused by the (anomalous) high temperature in Figure 11. Hence, for the reference simulation we only fitted the parameters using the observations in the first 55 weeks.

The selected results in Figure 12 confirm our expectations: the age at the beginning and end of the year is longer than in the middle, around week 25 . The age of a stem of about 40 days in the summer is in acccordance with our experience The age of 40 days in the summer is realistic, but teh afe of 90 days somewhat high; a realistic maximum is 70 days. We therefore hypothesieze that further optmization using data on the age of harvest stems is required. In such an improved optimization is probably necessary to vary parameter $K_{8}$ as well. 

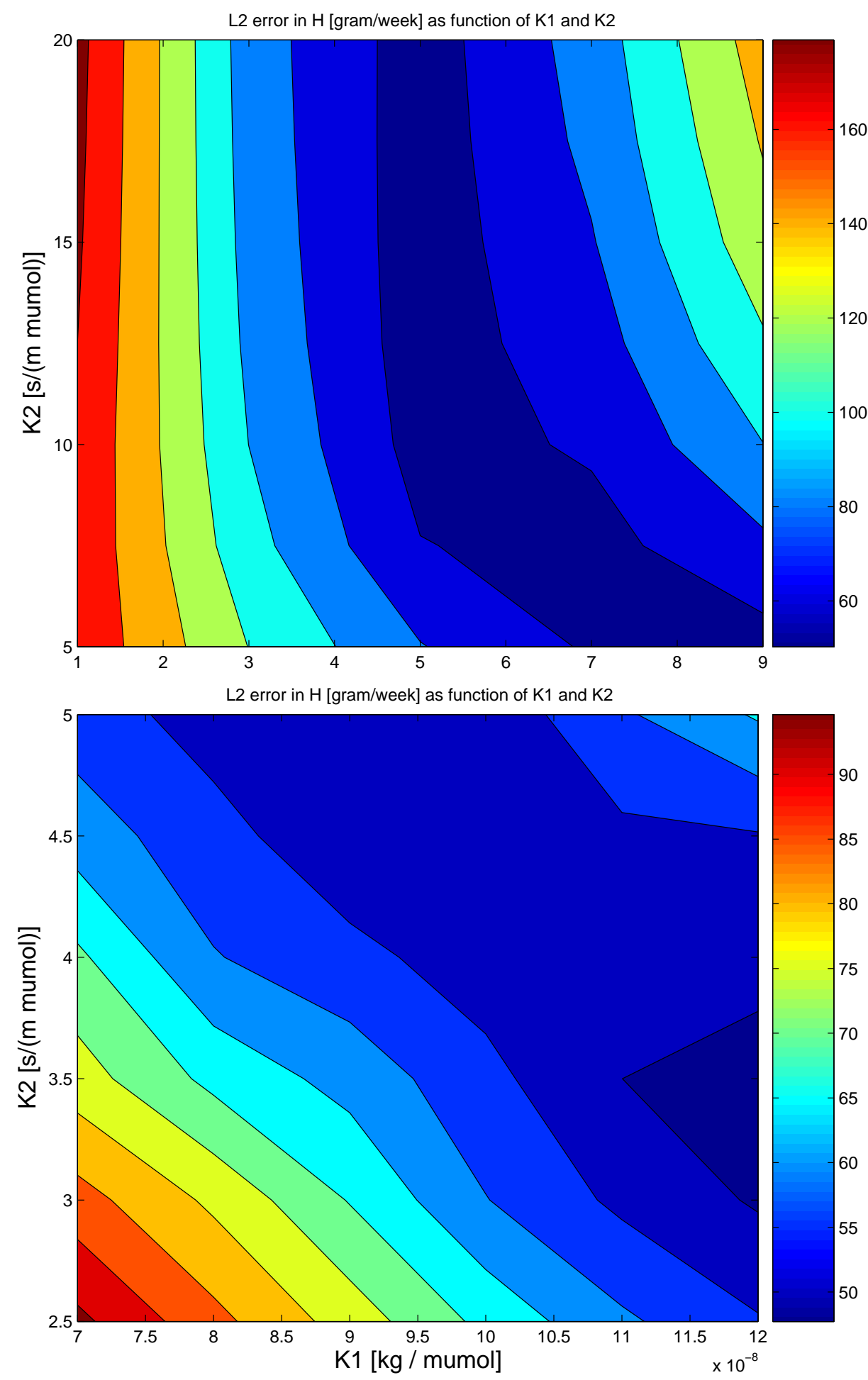

Figure 8: Parameter fits for $K_{1}$ and $K_{2}$ for reference simulations using all harvest data over 55 weeks. a) Minimum: $K_{1}=5.0 \times 10^{-8}, K_{2}=15$. b) Minimum: $K_{1}=$ $1.2 \times 10^{-7}, K_{2}=3.5$. 


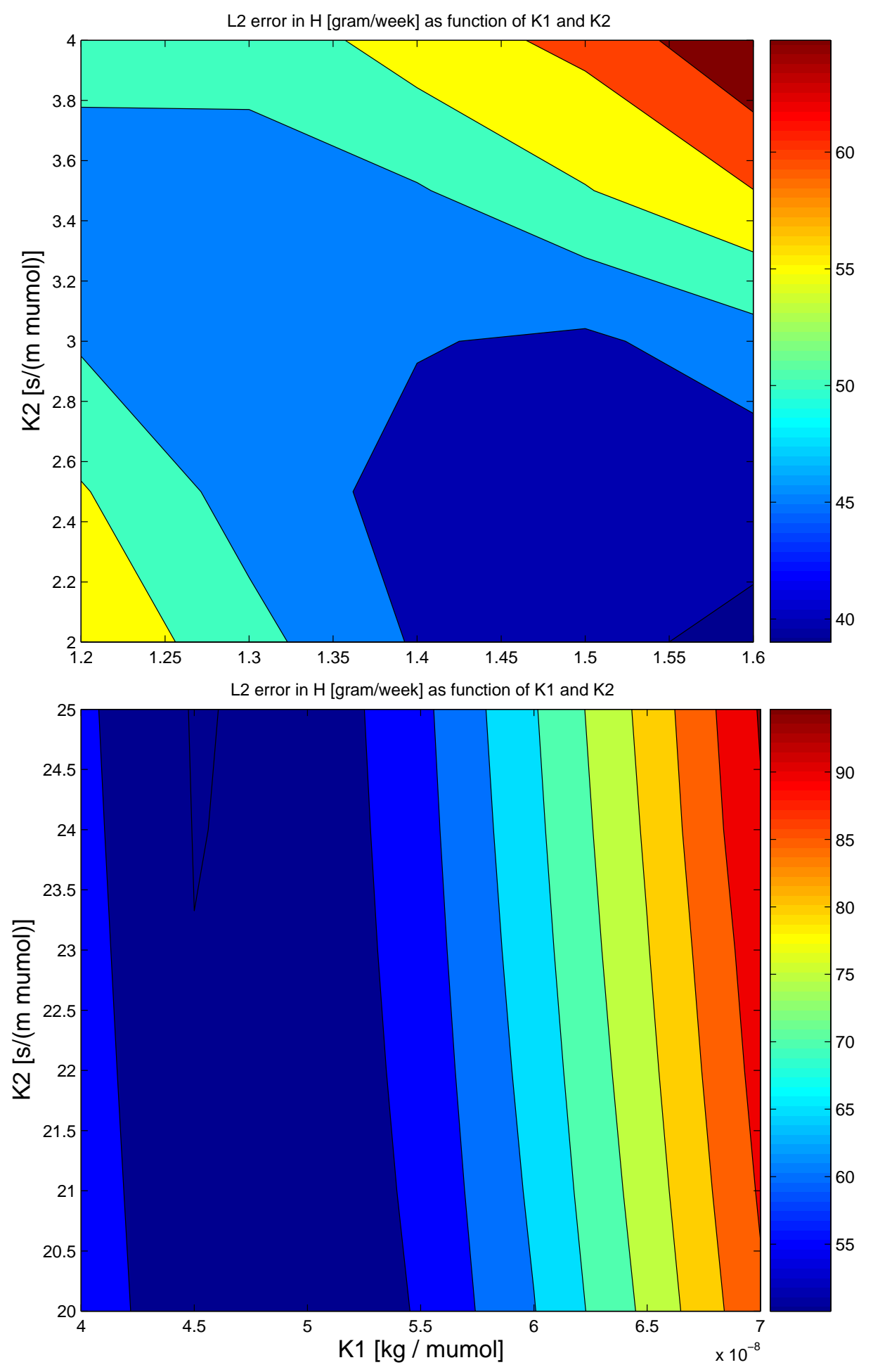

Figure 9: Parameter fits for $K_{1}$ and $K_{2}$ for reference simulations using all harvest data over 55 weeks. a) Minimum: $K_{1}=5.0 \times 10^{-8}, K_{2}=15$. b) Minimum: $K_{1}=$ $1.6 \times 10^{-7}, K_{2}=2$. 

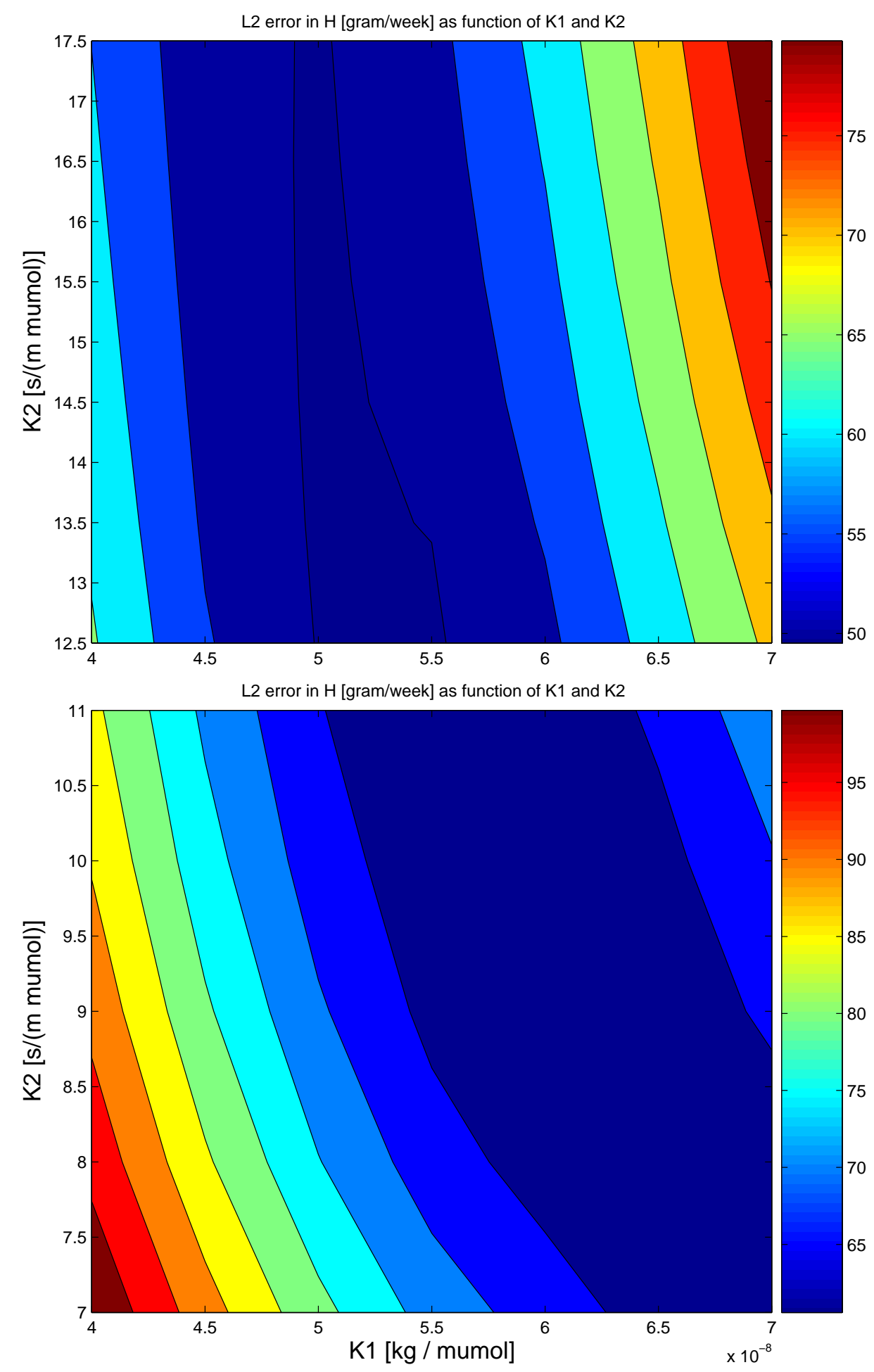

Figure 10: Parameter fits for $K_{1}$ and $K_{2}$ for the (a) reference simulation using all harvest data over 55 weeks with $K_{1}=5.0 \times 10^{-8}$ and $K_{2}=14.5$, and (b) the predictive simulation for the first half of the harvest data with $K_{1}=6 \times 10^{-8}$ and $K_{2}=10$, i.e. 28 weeks. 

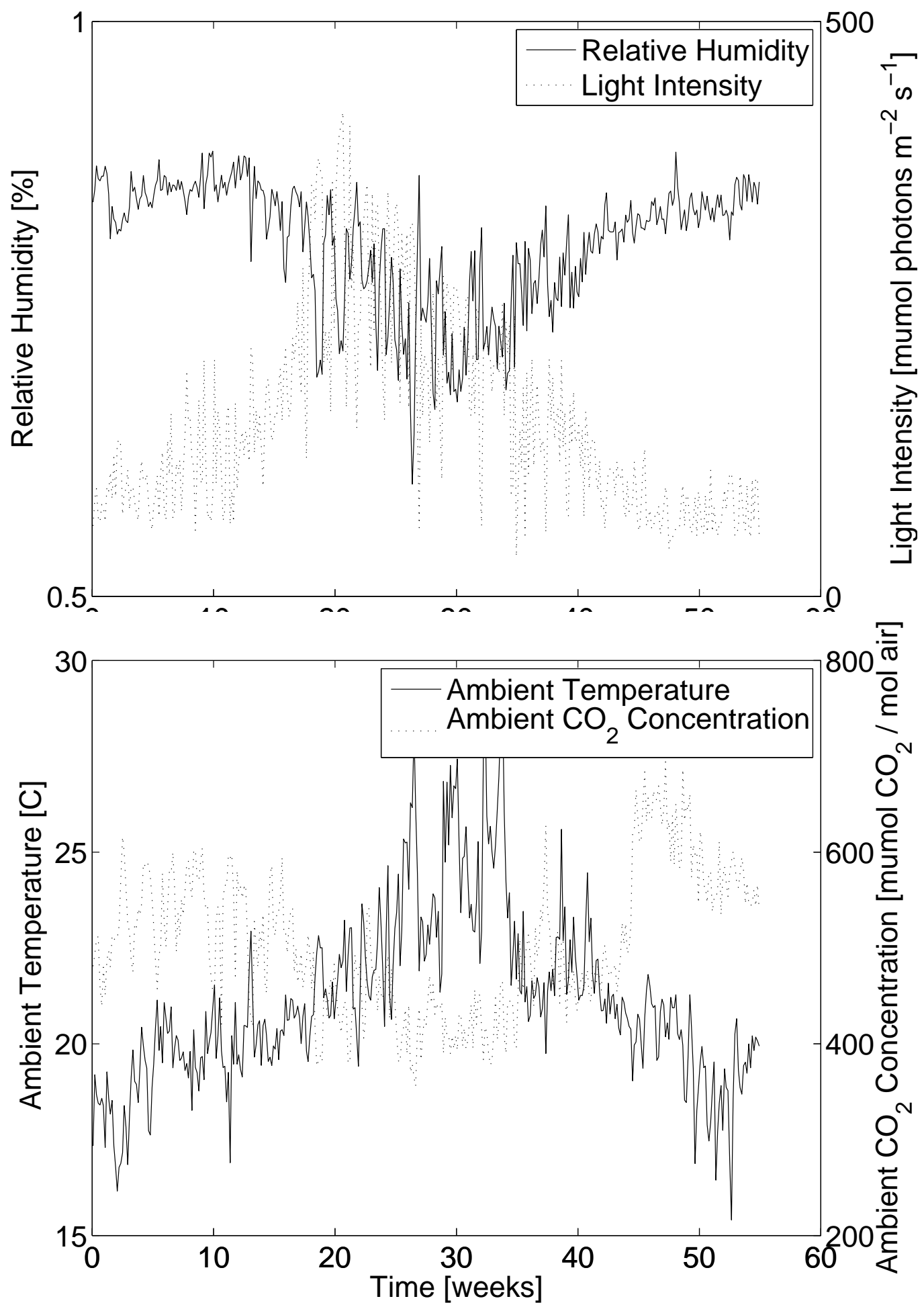

Figure 11: Daily averaged measurements of: (a) light intensity and relative humidity, and (b) ambient $\mathrm{CO}_{2}$ concentration and ambient temperature measurements. 

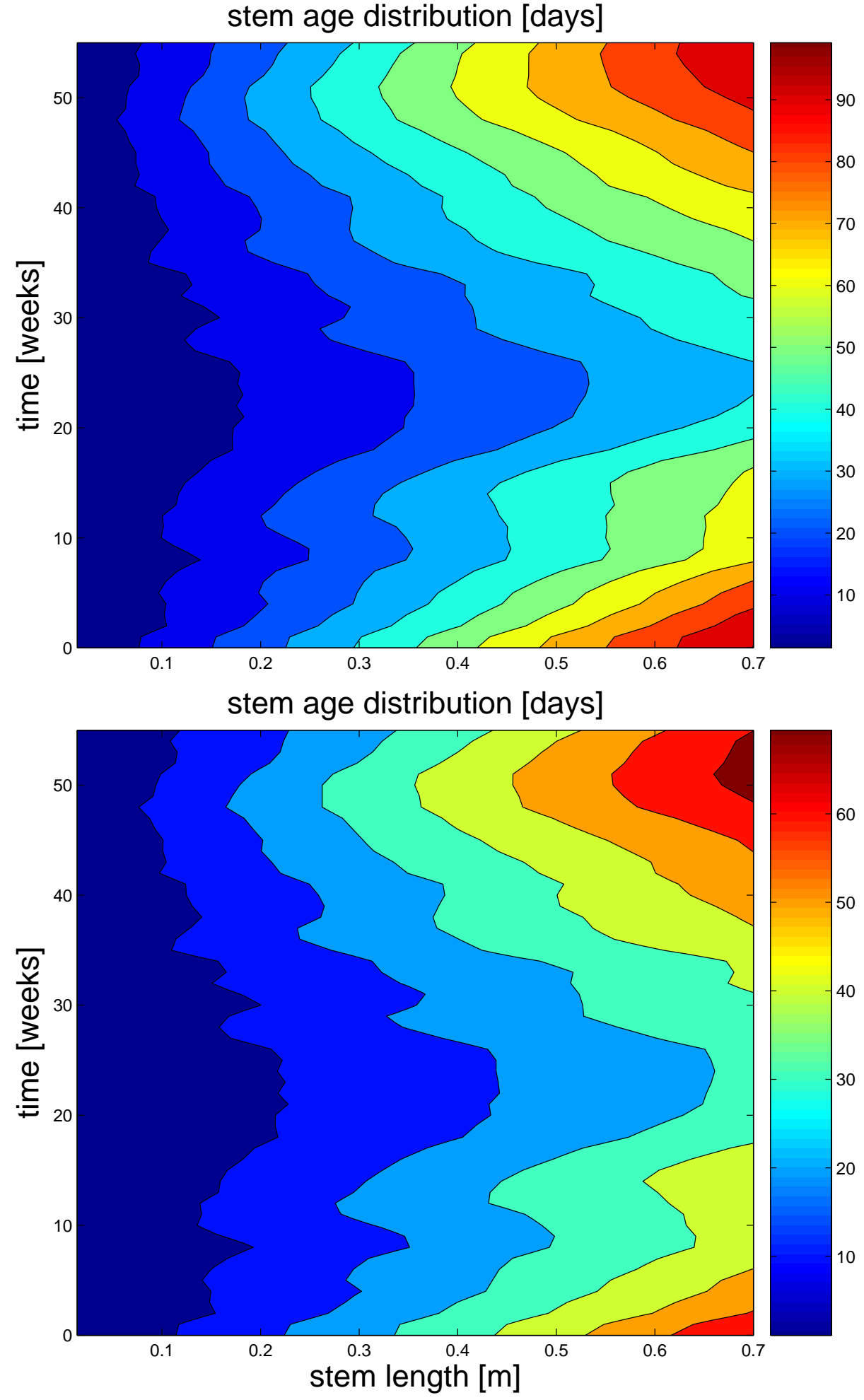

Figure 12: Stem age distribution as function of time and stem length for a) the reference simulation in which $K_{1}=5.0 \times 10^{-8}$ and $K_{2}=14.5$ are determined over a 55-week period, and b) the predictive simulation in which $K_{1}=6 \times 10^{-8}$ and $K_{2}=10$ are determined over the first 28 weeks. 

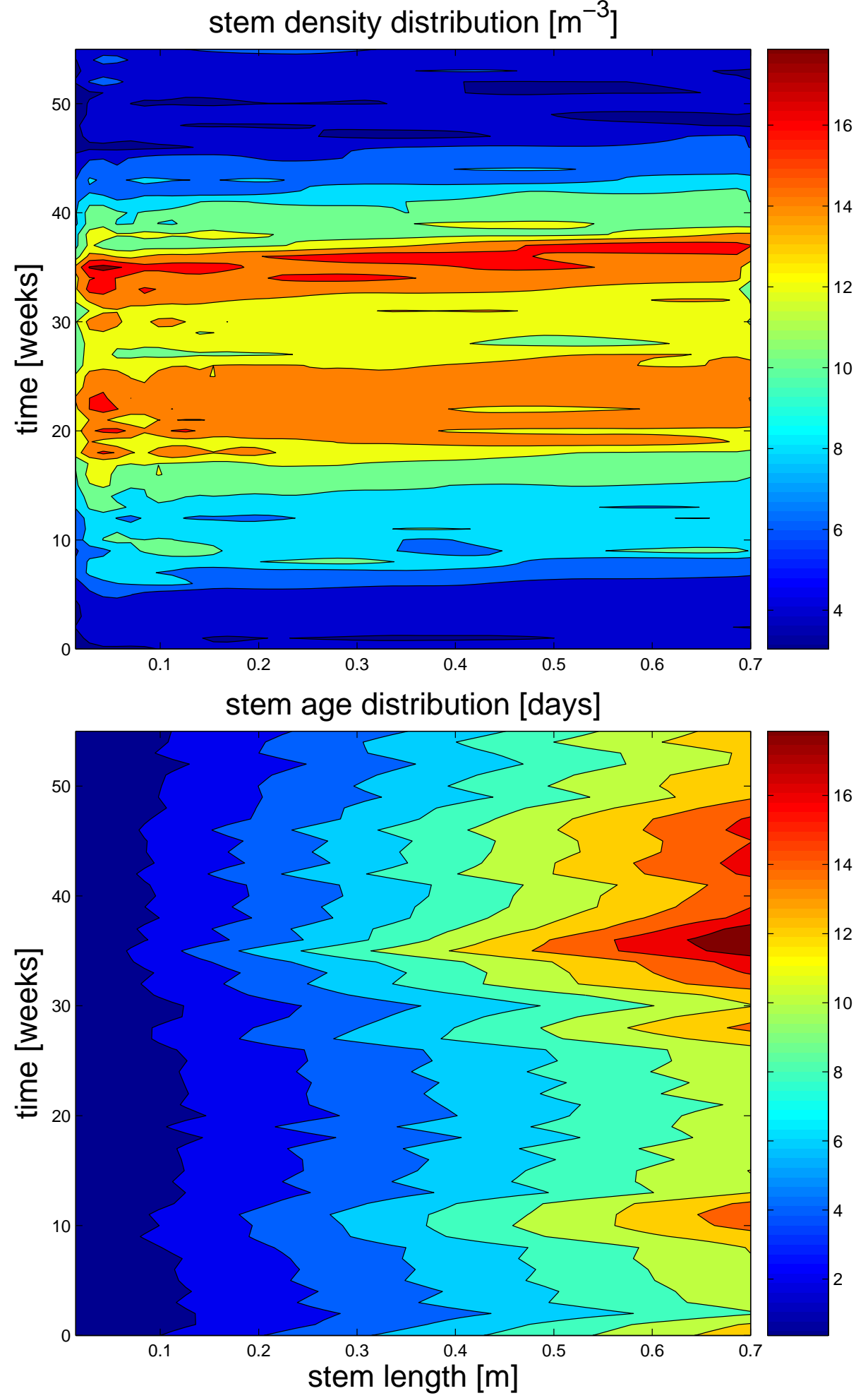

Figure 14: Stem density and age distribution as function of time and stem length for a 55 week optimization with $K_{1}=1.2 \times 10^{-7}, K_{2}=3.5$, see Figure 8 b). The results are anomalous because the stem age is to short and the stem density distribution unnatural. 